\title{
AgAu Nanotubes: Investigating the Effect of Surface Morphologies and Optical Properties over Applications in Catalysis and Photocatalysis
}

\author{
Thenner S. Rodrigues, Anderson G. M. da Silva, Arthur B. L. de Moura, \\ Rafael S. Geonmonond and Pedro H. C. Camargo*
}

\author{
Departamento de Química Fundamental, Instituto de Química, Universidade de São Paulo, \\ Av. Prof. Lineu Prestes, 748, 05508-000 São Paulo-SP, Brazil
}

\begin{abstract}
We herein report the preparation of AgAu nanotubes displaying controlled surface morphologies and optical properties by varying the reaction temperature during the galvanic reaction between $\mathrm{Ag}$ nanowires and $\mathrm{AuCl}_{4(\mathrm{aq})}^{-}$. As the $\mathrm{AgAu}$ nanotubes presented similar sizes and compositions, they enabled us to isolate the influence of surface morphology and optical properties over their catalytic and plasmonic photocatalytic activities towards methylene blue oxidation. At $25{ }^{\circ} \mathrm{C}$, $\mathrm{AgAu}$ nanotubes (AgAu 25) presented branched walls and surface plasmon resonance (SPR) band with low intensities in the visible were obtained. However, at $100{ }^{\circ} \mathrm{C}$, the AgAu nanotubes (AgAu 100) presented smooth surfaces and SPR bands that closely matched the emission spectra of a commercial halogen-tungsten lamp. The AgAu 25 nanotubes displayed better catalytic performances in classical heterogeneous catalysis as a result of its branched walls that lead to increased surface areas relative to the smooth nanotubes. Conversely, AgAu 100 nanotubes showed better activities in plasmonic photocatalysis due to its broader and more intense SPR bands. Thus, our results demonstrate the potential of the control over morphological and optical features towards the optimization of distinct catalytic phenomena.
\end{abstract}

Keywords: AgAu nanotubes, surface morphology, optical properties, catalysis, plasmonic photocatalysis

\section{Introduction}

Metal nanostructures have played a central role in several branches of science and technology including electronics,,${ }^{1,2}$ photonics, ${ }^{3-5}$ medicine, ${ }^{6,7}$ energy production, ${ }^{8,9}$ environmental remediation, ${ }^{10,11}$ and catalysis. ${ }^{12-15}$ Among various metallic nanomaterials, those based on gold $(\mathrm{Au})$ and silver $(\mathrm{Ag})$ have attracted special attention due to their fascinating optical properties in the visible range as a result of their localized surface plasmon resonance (LSPR) excitation. ${ }^{16,17}$ In addition to properties in sensing (enhanced spectroscopies), the LSPR excitation can also be put to work towards the promotion and/or enhancement of chemical processes, the so-called plasmonic catalysis that has emerged as a new frontier in the field of photocatalysis. ${ }^{18-20}$

Nanoparticles based on noble metals have also been widely employed in the field of heterogeneous catalysis and electrocatalysis due to their good stability and catalytic

*e-mail: camargo@iq.usp.br activities towards a wealth of transformations including oxidations, ${ }^{15,21-23}$ reductions,,${ }^{9,24}$ coupling reactions, ${ }^{25-28}$ among others. ${ }^{8,29-31}$ In these applications, it has been established that the precise control over their morphological and optical properties is a promising strategy to enhance/ optimize their performances..$^{29,32-37}$ Even though a large number of protocols have been described for the synthesis of plasmonic nanoparticles, new protocols that further enable the understanding and correlation of how catalytic responses are dependent on their morphological and optical features are needed to offer further insights into the rational design of catalysts with improved performances. Moreover, difficulties regarding their controlled synthesis in relatively larger scales still make their application in real catalytic and photocatalytic systems very challenging. ${ }^{35,38}$

We report herein the synthesis, in relatively large scales, of well-defined $\mathrm{AgAu}$ nanotubes displaying controlled and well-defined surface morphologies and optical properties. The AgAu nanotubes were obtained by the galvanic replacement reaction between $\mathrm{Ag}$ nanowires and $\mathrm{AuCl}_{4}{ }^{-}$ ions at 25 or $100{ }^{\circ} \mathrm{C}$. In this case, the reaction temperature 
control enabled us to maneuver the surface morphology and optical properties of the nanotubes in the visible range. The $\mathrm{AgAu}$ nanotubes were then employed as model catalysts towards the methylene blue oxidative degradation due to its importance in the field of environmental remediation. More specifically, regarding this transformation, the catalytic performance was investigated towards the "classical" heterogeneous catalysts and plasmonic photocatalysis. We focused on the understanding on how the catalytic and plasmonic photocatalytic activities of the AgAu nanotubes were dependent on their surface morphologies (branched or smooth surfaces) and optical properties (position and intensity of SPR extinction bands).

\section{Experimental}

Analytical grade chemicals silver nitrate $\left(\mathrm{AgNO}_{3}\right.$, 99\%, Sigma-Aldrich), polyvinylpyrrolidone (PVP, SigmaAldrich, M.W. 55,000 $\mathrm{g} \mathrm{mol}^{-1}$ ), polyvinylpyrrolidone (PVP, Sigma-Aldrich, M.W. 10,000 $\mathrm{g} \mathrm{mol}^{-1}$ ), ethylene glycol (EG, 99.8\%, Sigma-Aldrich), tetrachloroauric acid $\left(\mathrm{HAuCl}_{4} \cdot 3 \mathrm{H}_{2} \mathrm{O}, \geq 99.9 \%\right.$, Sigma-Aldrich), hydrochloric acid ( $\mathrm{HCl}, 37 \%$, Sigma-Aldrich), hydrogen peroxide (30 wt. $\%$ in $\mathrm{H}_{2} \mathrm{O}$, Sigma-Aldrich), tert-butyl hydroperoxide (70 wt. $\%$ in $\mathrm{H}_{2} \mathrm{O}$, Sigma-Aldrich) and methylene blue hydrate $\left(\mathrm{C}_{16} \mathrm{H}_{18} \mathrm{ClN}_{3} \mathrm{~S} \bullet \mathrm{xH}_{2} \mathrm{O}, \geq 95 \%\right.$, Sigma-Aldrich $)$ were used as received.

The scanning electron microscopy (SEM) images were obtained using a JEOL field emission gun electron microscope JSM6330F operated at $5 \mathrm{kV}$. The samples were prepared by drop-casting an aqueous suspension containing the nanostructures over a silicon wafer, followed by drying under ambient conditions. High-resolution transmission electron microscopy (HRTEM) images were obtained with a JEOL JEM2100 microscope operated at $200 \mathrm{kV}$. Energy dispersive X-ray (EDX) spectra were obtained in this instrument with an EDX detector configuration which has a total solid angle of ca. 0.13 srad. Samples for HRTEM were prepared by drop-casting an aqueous suspension of the nanostructures over a carbon-coated copper grid, followed by drying under ambient conditions. UV-Vis spectra were obtained from aqueous suspensions containing the nanostructures with a Shimadzu UV-1700 spectrophotometer. The Ag and $\mathrm{Au}$ atomic percentages were measured by inductively coupled plasma optical emission spectrometry (ICP OES) using a Spectro Arcos equipment. The X-ray diffraction (XRD) data were obtained using a Rigaku - Miniflex equipment, $\mathrm{CuK} \alpha$ radiation. The diffraction pattern was measured in the range of 10-90 degree with a 1 degree min $^{-1}$ angular speed scan.

\section{Synthesis of Ag nanowires}

In a typical procedure, $250 \mathrm{~mL}$ of $\mathrm{EG}$ was pre-heated for 1 hour at $140{ }^{\circ} \mathrm{C}$ with vigorous stirring, followed by the addition of $10 \mathrm{~mL}$ of $3 \mathrm{mmol} \mathrm{L}{ }^{-1} \mathrm{HCl}$ (in EG). After $10 \mathrm{~min}, 150 \mathrm{~mL}$ of $94 \mathrm{mmol} \mathrm{L}^{-1} \mathrm{AgNO}_{3}$ and $150 \mathrm{~mL}$ of $147 \mathrm{mmol} \mathrm{L}^{-1}$ PVP (both in EG) were simultaneously added dropwise to this mixture (at a speed of $45 \mathrm{~mL} \mathrm{~h}^{-1}$ ) and left overnight, yielding a beige suspension. The reaction flask was then allowed to cool down to room temperature.

Synthesis of AgAu nanotubes with controlled surface morphologies

In a typical procedure, a mixture containing $250 \mathrm{~mL}$ of PVP aqueous solution (0.1 wt.\%) and $50 \mathrm{~mL}$ of as prepared suspension containing the $\mathrm{Ag}$ wires was stirred at 25 or $100{ }^{\circ} \mathrm{C}$ for $10 \mathrm{~min}$ in a $1 \mathrm{~L}$ round-bottom flask. Then, $100 \mathrm{~mL}$ of a $0.6 \mathrm{mmol} \mathrm{L}^{-1} \mathrm{AuCl}_{4(\mathrm{aq})}^{-}$solution was added dropwise and the reaction allowed to proceed at 25 or $100{ }^{\circ} \mathrm{C}$ for $1 \mathrm{~h}$. After the syntheses, the reaction mixture volumes were reduced to $25 \mathrm{~mL}$ and washed twice with a $35 \mathrm{~g} \mathrm{~mL}^{-1} \mathrm{NaCl}_{(\mathrm{aq})}$ solution and three times with water by successive rounds of centrifugation at $7000 \mathrm{rpm}$ and removal of the supernatant. After washing, the AgAu nanomaterials were suspended in $400 \mathrm{~mL}$ of PVP aqueous solution (0.1 wt.\%) and storage at room temperature. For the catalytic applications, $10 \mathrm{~mL}$ of the $\mathrm{AgAu}$ nanotubes suspended in PVP aqueous solution (0.1 wt.\%) were washed twice with water by successive rounds of centrifugation at $7000 \mathrm{rpm}$ and removal of the supernatant and concentrated to $1 \mathrm{~mL}$ with water. After this step, the concentration of metal $(\mathrm{Ag}+\mathrm{Au})$ in the nanoparticles suspensions corresponded to $5.5 \mathrm{mmol} \mathrm{L}^{-1}$ (as measured by ICP OES).

\section{Photocatalytic oxidation of methylene blue}

Typically, $10 \mathrm{~mL}$ of a $10 \mathrm{mg} \mathrm{L}^{-1} \mathrm{MB}$ (methylene blue) aqueous solution and $1 \mathrm{~mL}$ of the suspension containing the washed catalysts were added in a $25 \mathrm{~mL}$ round-bottom flask. The resulting mixture was stirred for $1 \mathrm{~h}$ in the dark to achieve the adsorption and desorption equilibrium. Subsequently, the suspension was irradiated with a $300 \mathrm{~W}$ halogen tungsten lamp (OSRAM) mounted at a $2 \mathrm{~cm}$ distance from the glass reactor. During the experiments, $1 \mathrm{~mL}$ aliquots were taken and centrifuged at 15,000 rpm for 10 min to isolate the supernatant. Then, the UV-Vis spectra of the supernatant were measured as a function of time in the 500 to $750 \mathrm{~nm}$ range to probe the MB photodegradation. A calibration curve for absorbance as a function of the MB concentration was employed in order to monitor the 
transformation. The experiments employing hydrogen peroxide and tert-butyl hydroperoxide were performed by adding stoichiometric amount of oxidants necessary to the complete oxidation of methylene blue after $1 \mathrm{~h}$ of stirring in the dark, which correspond to 1.7 and $2.2 \mu \mathrm{L}$ of hydrogen peroxide and tert-butyl hydroperoxide, respectively.

\section{Results and Discussion}

Our studies started with the synthesis of Ag nanowires displaying well-defined shapes, smooth surfaces, and monodisperse sizes by a polyol approach ${ }^{39}$ as shown in Figure 1.

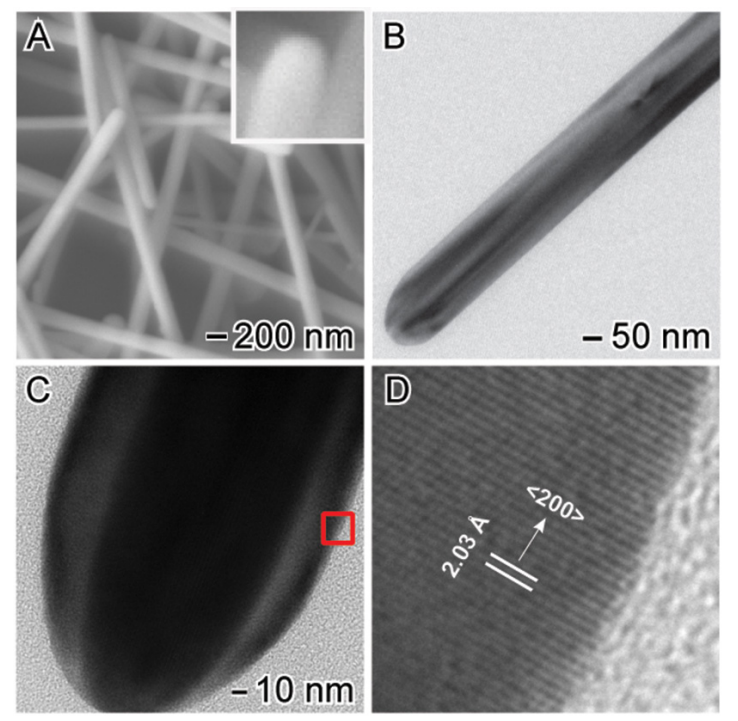

Figure 1. (A) SEM and (B-D) HRTEM images for Ag nanowires employed as starting materials for the synthesis of $\mathrm{AgAu}$ nanotubes. The image in (D) corresponds to phase contrast images of the area highlighted by the red square in $(\mathrm{C})$.

The obtained Ag nanowires were $155 \pm 15 \mathrm{~nm}$ in width and $>1 \mu \mathrm{m}$ in length. The histograms of size distribution (Figures S1A and S1B) indicate that the nanowires displayed a narrow size distribution, demonstrating the robustness of this approach for the synthesis of Ag nanowires in a large scale, which corresponds to an increase of 100 -folds compared to the conventional polyol protocol. ${ }^{39}$ Figures 1B-1D depict high-resolution transmission electron microscopy (HRTEM) images for the $\mathrm{Ag}$ nanowires, indicating that they present sharp tips (Figures 1B-1C) and are enclosed by $\{200\}$ sides facets with lattice spacings of $2.03 \AA$ of characteristic of fcc Ag (Figure 1D) in agreement with previous reports. ${ }^{39-41}$

As illustrated in Scheme 1, the strategy for the synthesis of AgAu nanotubes displaying controlled surface morphologies was based on the galvanic replacement reaction between $\mathrm{Ag}$ nanowires and $\mathrm{AuCl}_{4}^{-}$(aq) in the presence of PVP at different temperatures: 25 and $100^{\circ} \mathrm{C}$. Therefore, the nanotubes obtained in the synthesis carried out at 25 and $100{ }^{\circ} \mathrm{C}$ were denoted $\mathrm{AgAu} 25$ and 100, respectively.

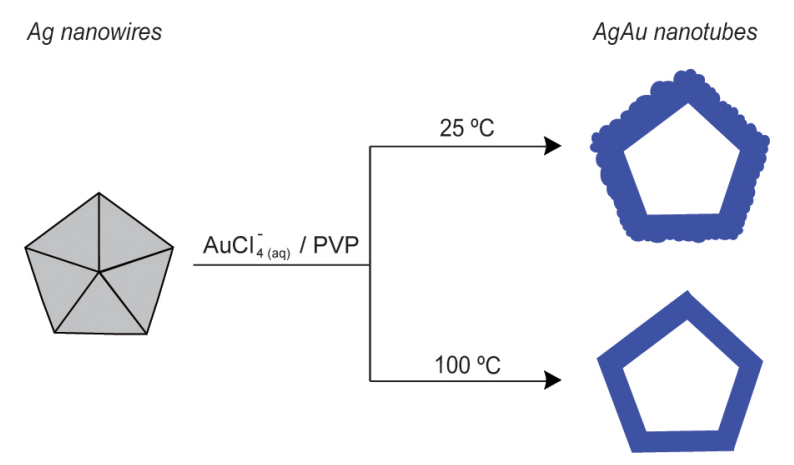

Scheme 1. Approach for the synthesis of AgAu nanotubes displaying controlled surface morphologies by galvanic replacement reaction between $\mathrm{Ag}$ nanowires and $\mathrm{AuCl}_{4(\text { (aq) }}^{-}$in the presence of PVP at different temperatures: 25 and $100{ }^{\circ} \mathrm{C}$.

At $25{ }^{\circ} \mathrm{C}, \mathrm{AgAu}$ nanotubes displaying branched surfaces. This occurs due to the precipitation of $\mathrm{AgCl}$ crystals (Kps ca. $1.8 \times 10^{-10}$ ) onto the surface of Ag during the galvanic replacement, which may act as templates for further Au deposition by an island-mode growth, which is associated with a lower rate of reconstruction processes such as Ostwald ripening and atomic diffusion. ${ }^{35,42-44}$ On the other hand, at $100^{\circ} \mathrm{C}$, no precipitation of $\mathrm{AgCl}$ crystals is observed, which drives the Au deposition favored by surface reconstruction/diffusion processes leading to the formation of hollow structures with smooth surfaces that replicate the surface morphology of the Ag nanowires used as templates. ${ }^{42,43,45}$ Thus, by employing this approach, it is possible to obtain $\mathrm{AgAu}$ nanotubes displaying distinct surface morphologies and thus systematically investigate this effect towards their optical, catalytic, and photocatalytic properties.

Figures 2A-2D show SEM (Figure 2A) and HRTEM (Figures 2B-2D) images for AgAu 25 nanotubes, while Figures 2E-2H show SEM (Figure 2E) and HRTEM (Figures 2F-2H) images for $\mathrm{AgAu} 100$ nanotubes.

It can be observed that both nanotubes displayed well-defined shapes and relatively monodisperse sizes (also depicted by their size distribution histograms in the Figures S1C-S1F). The elongated structures (from the Ag nanowires templates) was kept in both cases. However, it is very clear that the nanotubes displayed significant differences in their surface morphologies as a function of the temperature in which the galvanic reaction was performed. More specifically, at $25^{\circ} \mathrm{C} \mathrm{AgAu}$ nanotubes displayed branched surfaces, while nanotubes with smooth walls were obtained at $100^{\circ} \mathrm{C}$. Also, both $\mathrm{AgAu}$ nanotubes 

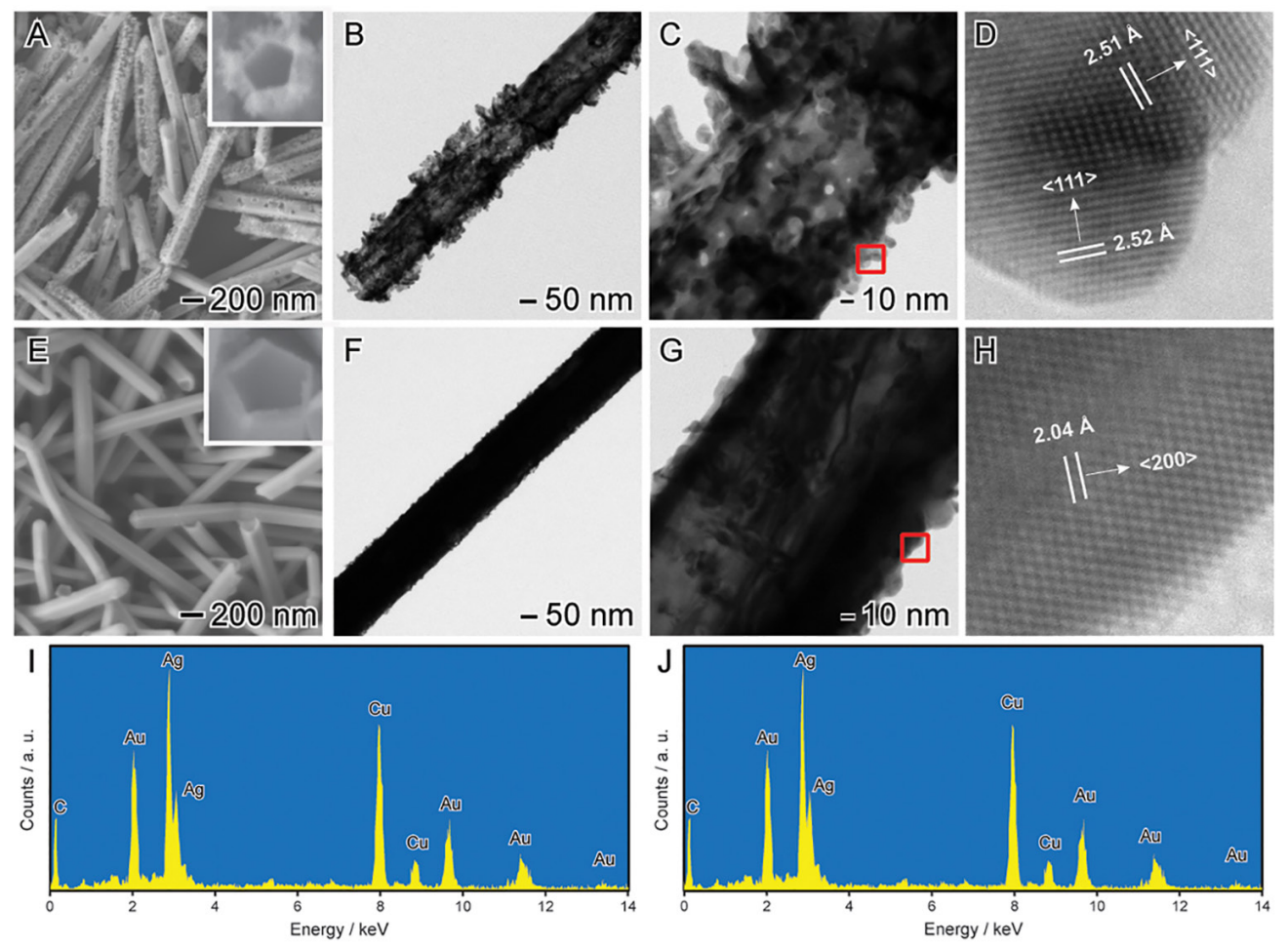

Figure 2. (A, E) SEM and (B-D, F-H) HRTEM images for (A-D) AgAu 25 and (E-H) AgAu 100 nanotubes. The images in (D) and (H) correspond to phase contrast images of the areas highlighted by the red squares in (C) and (G), respectively. (I) and (H) show EDX spectra obtained from individual (I) $\mathrm{AgAu} 25$ and (J) AgAu 100 nanotubes.

presented hollow interiors, which can be clearly visualized from the HRTEM images (Figures 2B-2C and 2F-2G) due to differences in mass-thickness contrast at the center of each nanotube. The $\mathrm{AgAu} 25$ and $\mathrm{AgAu} 100$ nanotubes were $178 \pm 15$ and $203 \pm 31 \mathrm{~nm}$ in width, respectively, and their shell thickness corresponded to 44 and $40 \mathrm{~nm}$, respectively. Interestingly, the HRTEM images for $\mathrm{AgAu}$ 25 and AgAu 100 (Figures 2D and 2H, respectively) showed that $\mathrm{AgAu} 25$ presented a polycrystalline surface comprised of crystalline islands that are randomly oriented as a consequence of the island-mode Au deposition at $25{ }^{\circ} \mathrm{C}$. However, $\mathrm{AgAu} 100$ displayed a surface enclosed by $\{200\}$ side facets similarly to the original Ag nanowires as a result of the Au epitaxial deposition over the surface of the Ag nanowires. ${ }^{42,43} \mathrm{EDX}$ spectra obtained from individual AgAu 25 (Figure 2I) and AgAu 100 (Figure 2J) nanotubes revealed that their composition corresponded to 30.0 and 26.6 at.\% in terms of $\mathrm{Au}$, respectively, in agreement with ICP OES results, which showed 26.7 and 27.2 at.\% in terms of $\mathrm{Au}$ for $\mathrm{AgAu} 25$ and $\mathrm{AgAu} 100$, respectively. The XRD patterns (Figure 3) confirmed the formation of $\mathrm{AgAu}$ nanostructures in both cases without the presence of any crystalline impurities.

However, as both fcc $\mathrm{Ag}$ and $\mathrm{Au}$ present a mismatch of only $0.17 \%$ in their lattice constants, ${ }^{46}$ the XRD diffraction peaks for these two species could not be resolved under our

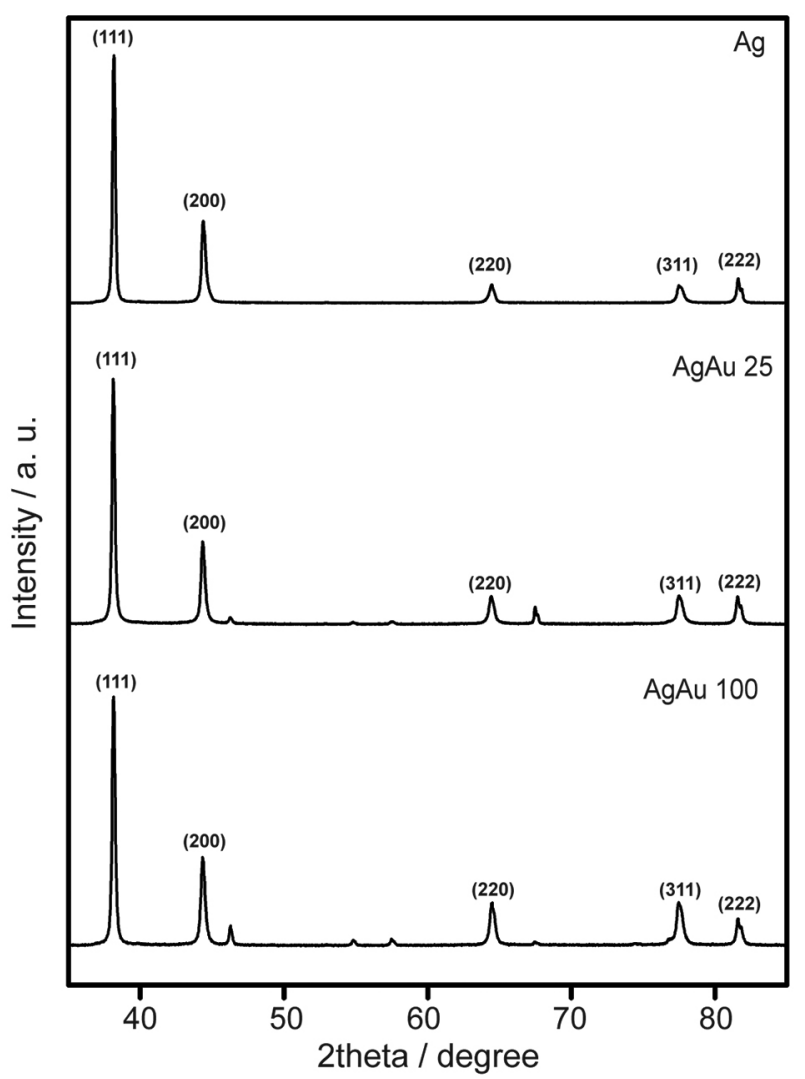

Figure 3. X-ray diffraction patterns recorded from the obtained $\mathrm{Ag}$ nanowires, $\mathrm{AgAu} 25$, and $\mathrm{AgAu} 100$ nanotubes. 
experimental conditions. Thus, the similar compositions, sizes, and wall thicknesses of the AgAu nanotubes enabled us to isolate the effect of the variation in the surface morphologies over their optical and catalytic properties. The optical properties of the AgAu nanotubes was investigated by UV-Vis spectroscopy (Figure 4).

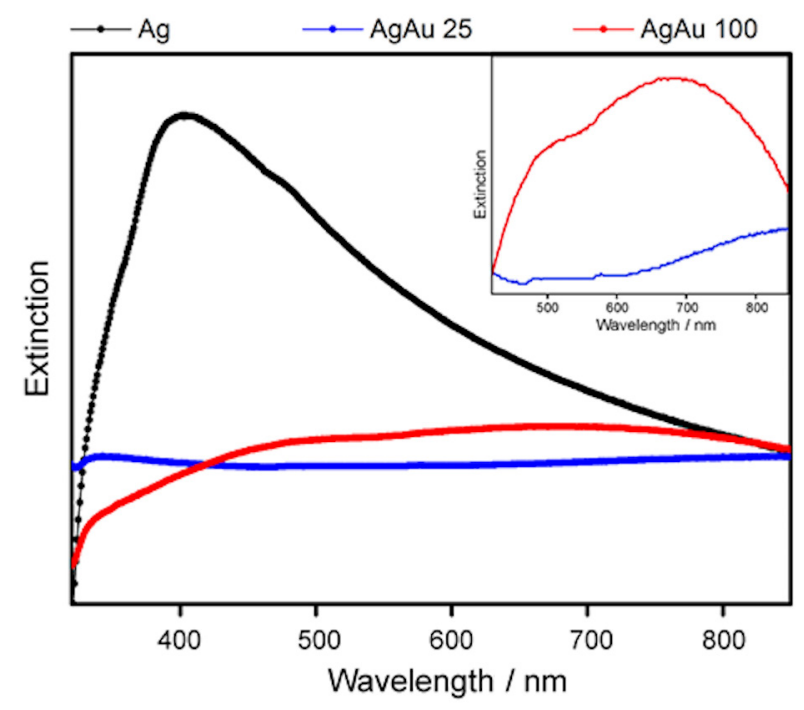

Figure 4. UV-Vis extinction spectra recorded from aqueous suspensions containing Ag nanowires (black trace), AgAu 25 (blue trace), and $\mathrm{AgAu} 100$ (red trace) nanotubes. The inset depicts a zoom in the 430-850 $\mathrm{nm}$ range for $\mathrm{AgAu}$ and $\mathrm{AgAu} 100$ nanotubes (color figure online).

Our results revealed the disappearance of the band associated to LSPR excitation in the Ag nanowires (black trace) at $405 \mathrm{~nm}$ in the nanotubes as a consequence of $\mathrm{Ag}$ dissolution from the template by the galvanic reaction with $\mathrm{AuCl}_{4}^{-}$(aq) ions together with the Au deposition. ${ }^{43,45,47-49} 35,39,40$ In addition, the SPR spectra for both AgAu nanotubes displayed significant red-shifts of their SPR bands relative to the Ag nanowires, which is in agreement with the Au deposition as well as the formation of the hollow interiors during the galvanic reaction. ${ }^{42,43}$ As depicted by the inset in the Figure 4, the AgAu 100 nanotubes displayed two SPR bands (one shoulder located at $510 \mathrm{~nm}$ and a broad signal centered at $690 \mathrm{~nm}$ ) with significantly higher intensities as compared the only broad band centered at $850 \mathrm{~nm}$ presented by the $\mathrm{AgAu} 25$.

It is important to note that the UV-Vis spectrum for $\mathrm{AgAu} 100$ nanotubes closely matches the emission spectra of a commercial halogen-tungsten lamp (Figure S2). Therefore, they represent potential candidates for improved efficiencies as plasmonic photocatalysis employing commercial halogen-tungsten lamp as the energy input. ${ }^{47,50}$ Conversely, due to the presence of small islands/branches at the surface as well as hollow interiors, the $\mathrm{AgAu} 25$ nanotubes represent a promising catalyst for application in conventional catalytic process (without light) as the branches/islands at the surface is expect to lead to increased surface areas relative to the smooth nanotubes. ${ }^{44,51}$ Therefore, after the synthesis of $\mathrm{AgAu}$ nanotubes displaying controlled surface morphologies and optical properties, we turned our attention for the investigation of their catalytic and photocatalytic properties towards an oxidative process. To this end, we employed methylene blue oxidative degradation as a model reaction due to its relevance in the field of environmental remediation..$^{52-54}$

Figures $5 \mathrm{~A}$ and $5 \mathrm{~B}$ show the kinetics profiles for methylene blue adsorption in the dark using different initial concentrations of this substrate in the presence of $\mathrm{AgAu} 25$ and $\mathrm{AgAu} 100$, respectively.
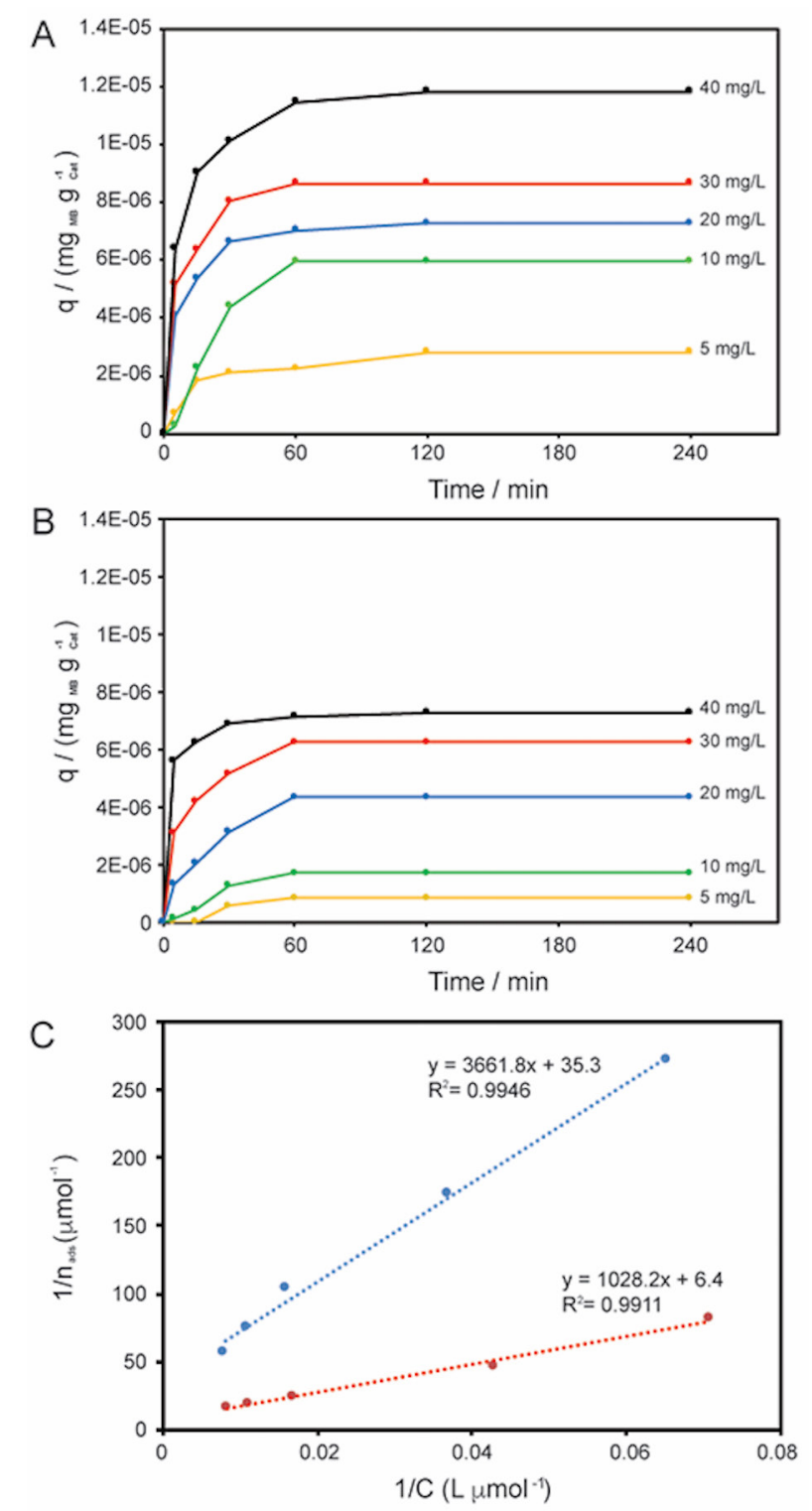

Figure 5. Kinetics of adsorption profiles of methylene blue in the dark in the presence of (A) AgAu 25 and (B) AgAu 100 nanotubes; (C) Langmuir linear transform of the curves in (A) and (B): AgAu 25 (blue trace) and $\mathrm{AgAu} 100$ (red trace) nanotubes (color figure online). 
Interestingly, for both $\mathrm{AgAu}$ nanotubes and for all employed initial concentrations of methylene blue, the equilibrium of adsorption/desorption was reached within $1 \mathrm{~h} .{ }^{55-57}$ However, in the presence of AgAu 25 (Figure 5A), the amount of methylene blue adsorbed per mass unit of catalyst (q) were significant higher for all concentrations as compared to AgAu 100 (Figure 5B). This behavior can be associated to the morphological features presented by $\mathrm{AgAu}$ 25 such as the presence of islands/branches at the surface that lead to increased surface areas relative to the smooth nanotubes. ${ }^{44,51}$ Figure $5 \mathrm{C}$ shows a linear transformation of the curves depicted in Figures 5A and 5B, which was determined by plotting the inverse of the number of mols of methylene blue adsorbed onto $\mathrm{AgAu}$ nanotubes $\left(1 / \mathrm{n}_{\mathrm{ads}}\right)$ as a function of final concentration of methylene blue in solution (C). Thus, according to the Langmuir model (equation 1), we can determine the constant of adsorption of methylene blue (k) for each $\mathrm{AgAu}$ nanotube under our employed conditions.

$\frac{1}{\mathrm{n}_{\mathrm{ads}}}=\frac{1}{\mathrm{n}_{0}}+\left(\frac{1}{\mathrm{n}_{0} \mathrm{k}}\right) \times \frac{1}{\mathrm{C}}$

Herein, the calculated adsorption constants for methylene blue $(\mathrm{k})$ corresponded to $9.75 \times 10^{-3}$ and $4.32 \times 10^{-3} \mathrm{~L} \mathrm{~mol}^{-1}$ for AgAu 25 and $\mathrm{AgAu} 100$, respectively, which is also in agreement with previous reports..$^{55}$

Figure 6 shows the methylene blue degradation employing different oxidizing agents under visible light
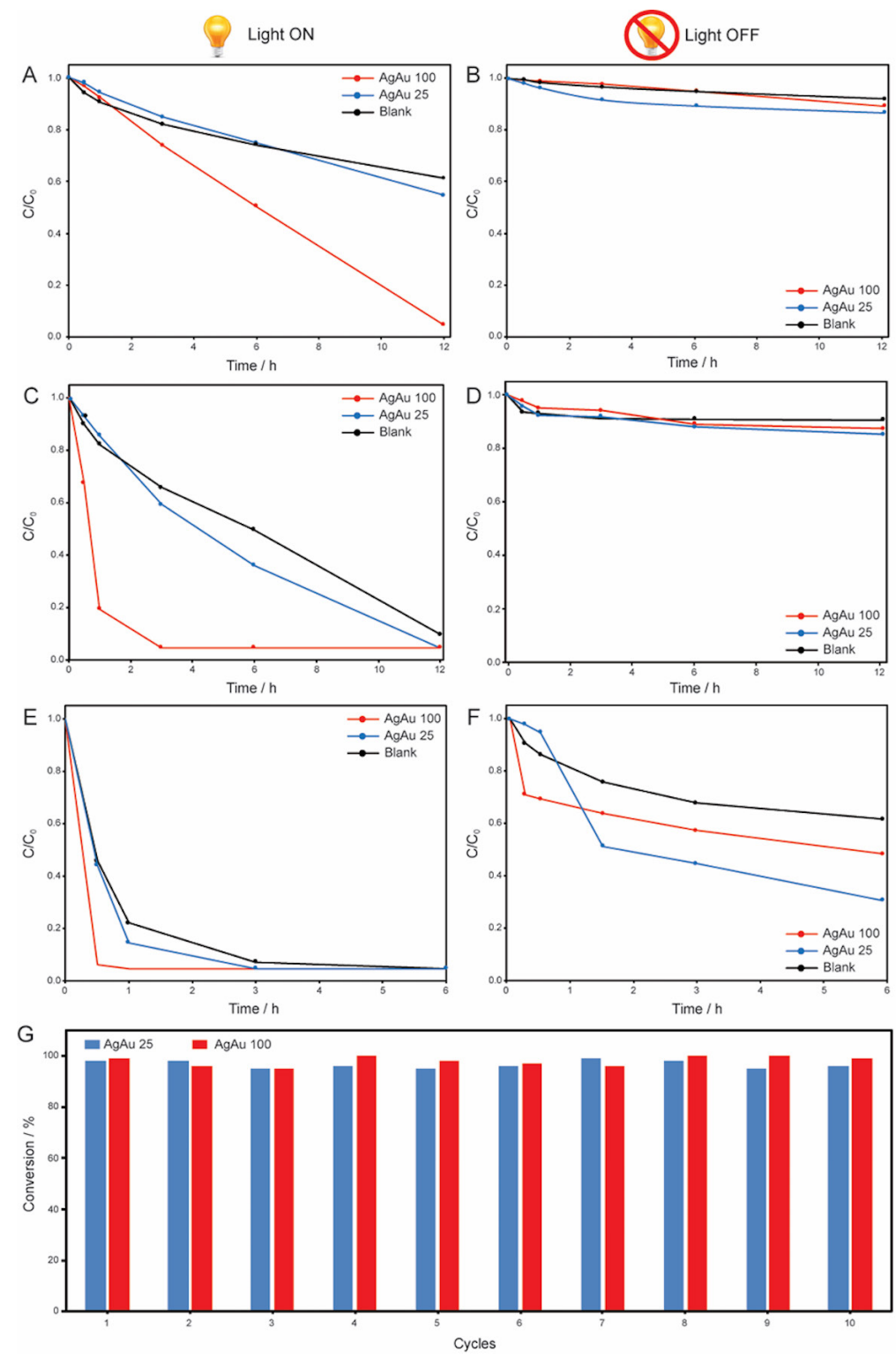

Figure 6. $C / C_{0}$ profiles as a function of time for the oxidation of methylene blue by (A-B) atmospheric air; (C-D) hydrogen peroxide; (E-F) and tert-butyl hydroperoxide employing $\mathrm{AgAu} 25$ (blue trace) and $\mathrm{AgAu} 100$ (red trace) nanotubes as catalysts. Blank reactions without any catalyst are shown as the black traces; (G) catalytic stability tests employing AgAu 25 and 100 nanotubes as catalysts (blue and red bars, respectively) (color figure online). 
illumination (left column) and without visible-light illumination (right column) using AgAu 25 (blue trace) and $\mathrm{AgAu} 100$ (red trace) as catalysts and a commercial halogen-tungsten lamp as the light source (left column).

For comparison, reactions without any catalyst (black trace) were also performed for all oxidizing systems. In all experiments, no organic degradation products were identified by gas chromatography mass spectrometry (GC-MS) analyses, which indicate the complete mineralization of methylene blue during the oxidation processes..$^{47,58,59} \mathrm{In}$ fact, as expected, a progressive increase in the methylene blue conversion was observed when stronger oxidizing agents were employed. More specifically, the conversion of methylene blue using both AgAu nanotubes as function of the oxidizing agent increased in the following order: atmospheric air (Figures 6A-6B) < hydrogen peroxide (Figures 6C-6D) < tert-butyl hydroperoxide (Figures 6E$6 \mathrm{~F})$. However, when the reactions were performed with and without illumination an interesting tendency was observed: without illumination (right column), the $\mathrm{AgAu} 25$ nanotubes showed a superior catalytic activity compared to $\mathrm{AgAu} 100$ in agreement with their larger surface area corroborated by the the adsorption experiments. However, under visible light illumination (left column), $\mathrm{AgAu} 100$ presented superior performances. This behavior can be associated with two very important features for photocatalytic processes presented by AgAu 100: (i) broader and more intense SPR bands in the visible range, and (ii) close matching between UV-Vis extinction spectrum for the photocatalyst and the emission spectra of the light source employed in the experiments. Thus, under the same experimental conditions, $\mathrm{AgAu}$ 100 tends to absorb more efficiently the light energy and enhance the photo-oxidative process in greater magnitude compared to $\mathrm{AgAu} 25 .{ }^{47}$ This results indicate that the control over the morphological and optical properties play a very important role in the optimization of catalytic and photocatalytic processes. Figure $6 \mathrm{G}$ shows the catalytic stability for the AgAu 25 (blue bars) and AgAu 100 (red bars) nanotubes towards methylene blue oxidation using tert-butyl hydroperoxide under visible light illumination (the most severe condition employed) expressed as a function of methylene blue conversion and the number of catalytic cycles. Interestingly, no significant loss of catalytic activity was detected even after 10 catalytic cycles for both materials. This result is in agreement with the SEM images for both materials after the catalytic experiments (Figure S3), which showed both $\mathrm{AgAu}$ nanotubes remained unchanged (without any aggregation or morphology loss), thus representing promising candidates for application as catalysts towards oxidation reactions without the need of a support to stabilize the catalyst.

\section{Conclusions}

In this paper, we systematically investigated the dependence of the the catalytic and plasmonic photocatalytic performances of $\mathrm{AgAu}$ nanotubes as a function of their surface morphologies and optical properties towards the oxidative degradation of methylene blue. We started by developing the synthesis of AgAu nanotubes having smooth or branched surfaces by controlling the temperature during the galvanic replacement reaction between $\mathrm{Ag}$ nanowires and $\mathrm{AuCl}_{4(\mathrm{aq})}^{-}$. This difference in surface morphologies also led to distinct optical properties (SPR extinction) in the visible range. Interestingly, the catalytic and photocatalytic performances of the $\mathrm{AgAu}$ nanotubes towards the methylene blue oxidative degradation showed a strong dependence on their morphological and optical properties. More specifically, AgAu 25 showed a superior catalytic performance without visible-light illumination due to its higher surface area as a consequence of its branched walls. However, $\mathrm{AgAu} 100$ showed superior activity in the experiments performed under visible light illumination due to its broader and more intense SPR bands which close matched the emission spectrum of the light source employed in the photocatalytic reactions. These results show that surface morphologies and optical properties play an important and distinct role over the optimization of the catalytic or plasmonic photocatalytic properties.

\section{Supplementary Information}

Supplementary information is available free of charge at http://jbcs.sbq.org.br as a PDF file.

\section{Acknowledgments}

This work was supported by the Fundação de Amparo à Pesquisa do Estado de São Paulo (FAPESP) (grant number 2015/21366-9). P. H. C. C. thanks the CNPq for the research fellowships. A. G. M. S. thanks the CNPq and T. S. R. and R. S. G. thank CAPES for the fellowships.

\section{References}

1. Shen, W.; Zhang, X.; Huang, Q.; Xu, Q.; Song, W.; Nanoscale 2014, 6, 1622.

2. Minari, T.; Kanehara, Y.; Liu, C.; Sakamoto, K.; Yasuda, T.; Yaguchi, A.; Tsukada, S.; Kashizaki, K.; Kanehara, M.; Adv. Funct. Mater. 2014, 24, 4869.

3. Clavero, C.; Nat. Photonics 2014, 8, 95.

4. Zhang, Z.; Zhang, L.; Hedhili, M. N.; Zhang, H.; Wang, P.; Nano Lett. 2013, 13, 14. 
5. Singh, A.; Kim, W.; Kim, Y.; Jeong, K.; Kang, C. S.; Kim, Y.; Koh, J.; Mahajan, S. D.; Prasad, P. N.; Kim, S.; Adv. Funct. Mater. 2016, 39, 7057.

6. Lane, L. A.; Qian, X.; Nie, S.; Chem. Rev. 2015, 115, 10489.

7. Lohse, S. E.; Murphy, C. J.; J. Am. Chem. Soc. 2012, 134, 15607.

8. Zeng, Z.; Tan, C.; Huang, X.; Bao, S.; Zhang, H.; Energy Environ. Sci. 2014, 7, 797.

9. Zhao, X.; Long, R.; Liu, D.; Luo, B.; Xiong, Y.; J. Mater. Chem. A 2015, 3, 9390 .

10. Wu, L.; Li, F.; Xu, Y.; Zhang, J. W.; Zhang, D.; Li, G.; Li, H.; Appl. Catal., B 2015, 164, 217.

11. Khin, M. M.; Nair, A. S.; Babu, V. J.; Murugan, R.; Ramakrishna, S.; Energy Environ. Sci. 2012, 5, 8075.

12. Linic, S.; Christopher, P.; Xin, H.; Marimuthu, A.; Acc. Chem. Res. 2013, 46, 1890.

13. Alves, A. K.; Berutti, F. A.; Sánchez, F. A. L. In Nanomaterials and Catalysis; Bergmann, C. P.; de Andrade, M. J., eds.: Springer-Verlag: Berlin, Germany, 2011, p. 93.

14. Polshettiwar, V.; Varma, R. S.; Green Chem. 2010, 12, 743.

15. Freakley, S. J.; He, Q.; Kiely, C. J.; Hutchings, G. J.; Catal. Lett. 2015, 145, 71.

16. Sun, Y.; Xia, Y.; Analyst 2003, 128, 686.

17. Huang, Y.-F.; Zhang, M.; Zhao, L.-B.; Feng, J.-M.; Wu, D.-Y.; Ren, B.; Tian, Z.-Q.; Angew. Chem., Int. Ed. 2014, 53, 2353.

18. Wang, P.; Huang, B.; Dai, Y.; Whangbo, M.-H.; Phys. Chem. Chem. Phys. 2012, 14, 9813.

19. Hou, W.; Cronin, S. B.; Adv. Funct. Mater. 2013, 23, 1612.

20. Linic, S.; Aslam, U.; Boerigter, C.; Morabito, M.; Nat. Mater. 2015, 14, 567.

21. Christopher, P.; Xin, H.; Linic, S.; Nat. Chem. 2011, 3, 467.

22. da Silva, A. M.; Rodrigues, T.; Taguchi, L. K.; Fajardo, H.; Balzer, R.; Probst, L. D.; Camargo, P. C.; J. Mater. Sci. 2015, 1.

23. Smolentseva, E.; Costa, V. V; Cotta, R. F.; Simakova, O.; Beloshapkin, S.; Gusevskaya, E. V; Simakov, A.; ChemCatChem 2015, 7, 1011.

24. Fang, J.; Li, J.; Zhang, B.; Yuan, X.; Asakura, H.; Tanaka, T.; Teramura, K.; Xie, J.; Yan, N.; Nanoscale 2015, 7, 6325.

25. da Silva, A. G. M.; Rodrigues, T. S.; Correia, V. G.; Alves, T. V.; Alves, R. S.; Ando, R. A.; Ornellas, F. R.; Wang, J.; Andrade, L. H.; Camargo, P. H. C.; Angew. Chem., Int. Ed. 2016, 55, 7111.

26. Wang, F.; Li, C.; Chen, H.; Jiang, R.; Sun, L.-D.; Li, Q.; Wang, J.; Yu, J. C.; Yan, C.-H.; J. Am. Chem. Soc. 2013, 135, 5588.

27. Fihri, A.; Bouhrara, M.; Nekoueishahraki, B.; Basset, J.-M.; Polshettiwar, V.; Chem. Soc. Rev. 2011, 40, 5181.

28. Guha, N. R.; Sharma, S.; Bhattacherjee, D.; Thakur, V.; Bharti, R.; Reddy, C. B.; Das, P.; Green Chem. 2016, 18, 1206.

29. Wang, X.; Figueroa-Cosme, L.; Yang, X.; Luo, M.; Liu, J.; Xie, Z.; Xia, Y.; Nano Lett. 2016, 16, 1467.

30. Hu, P.; Song, Y.; Chen, L.; Chen, S.; Nanoscale 2015, 7, 9627.

31. Liu, Q.; Xu, Y.-R.; Wang, A.-J.; Feng, J.-J.; J. Power Sources 2016, 302, 394.
32. da Silva, A. G. M.; Rodrigues, T. S.; Wang, J.; Yamada, L. K.; Alves, T. V.; Ornellas, F. R.; Ando, R. A.; Camargo, P. H. C.; Langmuir 2015, 31, 10272.

33. da Silva, A. G. M.; Rodrigues, T. S.; Taguchi, L. S. K.; Fajardo, H. V.; Balzer, R.; Probst, L. F. D.; Camargo, P. H. C.; J. Mater. Sci. 2016, 51, 603.

34. Xu, R.; Wang, D.; Zhang, J.; Li, Y.; Chem. - Asian J. 2006, 1, 888.

35. Rodrigues, T. S.; da Silva, A. H. M.; da Silva, A. G. M.; Ceara, D. G.; Gomes, J. F.; Assaf, J. M.; Camargo, P. H. C.; Catal. Sci. Technol. 2016, 6, 2162.

36. Chanda, K.; Rej, S.; Huang, M. H.; Chem. - Eur. J. 2013, 19, 16036.

37. Tsai, Y.-H.; Chanda, K.; Chu, Y.-T.; Chiu, C.-Y.; Huang, M. H.; Nanoscale 2014, 6, 8704.

38. Stark, W. J.; Stoessel, P. R.; Wohlleben, W.; Hafner, A.; Chem. Soc. Rev. 2015, 44, 5793.

39. Chen, J.; Wiley, B. J.; Xia, Y.; Langmuir 2007, 23, 4120.

40. Sun, Y.; Gates, B.; Mayers, B.; Xia, Y.; Nano Lett. 2002, $2,165$.

41. Wiley, B.; Sun, Y.; Mayers, B.; Xia, Y.; Chem. - Eur. J. 2005, 11,454

42. Sun, Y.; Mayers, B. T.; Xia, Y.; Nano Lett. 2002, 2, 481.

43. Sun, Y.; Mayers, B.; Xia, Y.; Adv. Mater. 2003, 15, 641.

44. Xia, X.; Wang, Y.; Ruditskiy, A.; Xia, Y.; Adv. Mater. 2013, 25 , 6313.

45. Sun, Y.; Mayers, B.; Herricks, T.; Xia, Y.; Nano Lett. 2003, 3, 955.

46. Foiles, S. M.; Baskes, M. I.; Daw, M. S.; Phys. Rev. B 1986, 33, 7983.

47. Rodrigues, T. S.; da Silva, A. G. M.; de Moura, A. B. L.; Freitas, I. G.; Camargo, P. H. C.; RSC Adv. 2016, 6, 62286.

48. Skrabalak, S. E.; Chen, J.; Sun, Y.; Lu, X.; Au, L.; Cobley, C. M.; Xia, Y.; Acc. Chem. Res. 2008, 41, 1587.

49. Jang, H.-J.; Ham, S.; Acapulco, J. A. I.; Song, Y.; Hong, S.; Shuford, K. L.; Park, S.; J. Am. Chem. Soc. 2014, 136, 17674.

50. Verbruggen, S. W.; Keulemans, M.; Filippousi, M.; Flahaut, D.; Van Tendeloo, G.; Lacombe, S.; Martens, J. A.; Lenaerts, S.; Appl. Catal., B 2014, 156-157, 116.

51. Rodrigues, T. S.; da Silva, A. G. M.; Gonçalves, M. C.; Fajardo, H. V.; Balzer, R.; Probst, L. F. D.; Camargo, P. H. C.; ChemNanoMat 2015, 1, 46.

52. Paul, B.; Bhuyan, B.; Dhar Purkayastha, D.; Dey, M.; Dhar, S. S.; Mater. Lett. 2015, 148, 37.

53. Zhou, G.; Fang, F.; Chen, Z.; He, Y.; Sun, H.; Shi, H.; Catal. Commun. 2015, 62, 71.

54. Naraginti, S.; Stephen, F. B.; Radhakrishnan, A.; Sivakumar, A.; Spectrochim. Acta, Part A 2015, 135, 814.

55. Houas, A.; Lachheb, H.; Ksibi, M.; Elaloui, E.; Guillard, C.; Herrmann, J.-M.; Appl. Catal., B 2001, 31, 145.

56. Shahabuddin, S.; Muhamad Sarih, N.; Mohamad, S.; Joon Ching, J.; Polymers 2016, 8, 27. 
57. Matos, J.; Fierro, V.; Montaña, R.; Rivero, E.; de Yuso, A. M.; Zhao, W.; Celzard, A.; Appl. Catal., A 2016, 517, 1.

58. Sahoo, C.; Gupta, A. K.; J. Environ. Sci. Health, Part A: Toxic/ Hazard. Subst. Environ. Eng. 2015, 50, 1333.
59. Lin, J.; Weng, X.; Jin, X.; Megharaj, M.; Naidu, R.; Chen, Z.; RSC Adv. 2015, 5, 70874.
Submitted: September 30, 2016

Published online: November 22, 2016 